\title{
INSTITUCIONALIZAÇÃO DE NÚCLEOS DE INOVAÇÃO TECNOLÓGICA EM INSTITUIÇÕES DE CIÊNCIA E TECNOLOGIA DA REGIÃO SUL DO BRASIL ${ }^{1}$
}

\author{
Hilka Pelizza Vier Machado ${ }^{2}$ \\ Rejane Sartori ${ }^{3}$ \\ João Marcelo Crubellate ${ }^{4}$
}

http://dx.doi.org/10.1590/1413-2311.177.67190

\begin{abstract}
RESUMO
No contexto brasileiro, a Lei de Inovação, promulgada em 2004 e reiterada pelo Marco Legal da Inovação em 2016, estabeleceu que as Instituições de Ciência e Tecnologia (ICTs) nacionais dispusessem de Núcleos de Inovação Tecnológica (NITs) para gerir suas respectivas políticas de inovação. A institucionalização, abordagem que explica como e porque estruturas e processos tornam-se legitimados, compreende um conjunto de componentes chaves, fundamentados na habitualização, que representa a formalização de arranjos em políticas e procedimentos. Nesse sentido, esta pesquisa teve como objetivo conhecer como ocorreu a institucionalização de NITs implantados no sul do Brasil, uma vez que estes representam importante pilar no Sistema Nacional de Ciência, Tecnologia e Inovação (SNCT\&I). A fim de atingir o objetivo proposto, foi realizada uma pesquisa qualitativa e exploratória, com dados secundários extraídos de relatórios do Formulário para Informações sobre a Política de Propriedade Intelectual das ICTS do Brasil (Formict), e dados primários advindos de questionário aplicado a coordenadores de NITs de três estados do sul do país. Foram empregadas técnicas usuais da análise de conteúdo para codificação e interpretação dos dados. Os resultados mostraram algumas dificuldades no processo de institucionalização dos NITs, principalmente vinculadas ao não surgimento de uma cultura empreendedora, a pouca autonomia e aos entraves burocráticos na contratação de pessoal, evidenciados no modelo de organização do campo, baseado em um pilar institucional regulativo. Conclui-se que os NITs se encontram em fase de habitualização, sendo que o estágio de sedimentação e o processo de institucionalização variam conforme a instituição, embora tenha sido observado mimetismo nas ações.
\end{abstract}

Palavras Chave: Inovação. Sistema Nacional de Inovação. Núcleos de Inovação Tecnológica. Transferência de Tecnologia.

\section{INSTITUCIONALIZATION OF UNITS OF TECHNOLOGICAL INNOVATION IN INSTITUTIONS OF SCIENCE AND TECHNOLOGY OF THE SOUTH AREA OF BRAZIL}

\footnotetext{
${ }^{1}$ Recebido em 21/08/2016; aprovado em 18/07/2017.

${ }^{2}$ Centro Universitário UniCesumar e Universidade do Oeste de Santa Catarina - hilkavier@yahoo.com.

${ }^{3}$ Centro Universitário UniCesumar e Universidade Estadual de Maringá - rejanestr@ gmail.com.

${ }^{4}$ Universidade Estadual de Maringá - jmcrubellate@uem.br.
} 


\begin{abstract}
In the Brazilian context, the Law of Innovation, promulgated in 2004 and reiterated by the Legal Marco of the Innovation in 2016, established that the Institutions of Science and National Technology (ICTs) should have Nuclei of Technological Innovation (NITs) to manage their respective innovation politics. The institutionalization, approach that explains how and why structure and processes are legitimated, includes a group of key components based in the habitualization, which represents the formalization of politics and procedures arrangements. In that sense, this research had as objective to know how the institutionalization of NITs located in Southern Brazil happened, because they represent important pillars in the National System of Science, Technology and innovation (SNCT\&I). To reach the proposed objective, a qualitative and exploratory research was developed, with secondary data of the Form for Information on the Politics of Intellectual Property of ICTS of Brazil (Formict), and primary data of questionnaires answered by coordinators of NITs of three Southern Brazil states. Content analysis was used for coding and interpretation of data. The results showed some difficulties in the process of institutionalization of NITs, mainly linked to the absence of an entrepreneurial culture, little autonomy and the bureaucratic impediments in personnel's recruiting, evidencing a model of organization based on an institutional regulative pillar. The conclusion is that NITs are in the habitualization phase, sedimentation stage and the institutionalization process vary according to the institution, although mimetism has been observed in their actions.
\end{abstract}

Keywords: Innovation. National System of Innovation. Nuclei of Technological Innovation. Technology Transference.

\title{
INSTITUCIONALIZACIÓN DE NÚCLEOS DE LA INNOVACIÓN TECNOLÓGICA EN LAS INSTITUCIONES DE CIENCIA Y TECNOLOGÍA DEL ÁREA DE SUR DE BRASIL
}

\section{RESUMEN}

En el contexto brasileño, la ley de la innovación fue promulgada en 2004 y reiterada por Marco legal de la innovación en 2016, determinando que las Instituciones de Ciencia y Tecnología (ICTs) tendrían Núcleos de Innovación Tecnológica (NITs) para ejecutar su respectiva política de innovación. La institucionalización, el enfoque que explica cómo y porque estructuras y procesos son legitimados, comprende un grupo de componentes ubicado en el habitualización, que representa la formación de arreglos en la política y en los procedimientos. Esta investigación teuve como objetivo saber cómo ocurrió el proceso de institucionalización de NITs en el sur de Brasil, una vez que éstos corresponden a un pilar importante en el Sistema Nacional de Ciencia, Tecnología e Innovación (SNCT \& I). Para llegar al objetivo propuesto, se realizó una investigación cualitativa y exploratoria. Los datos secundarios fueron extraídos de informes del Formulario para la Información sobre la Política de 1 Propiedad Intelectual de ICTS de Brasil (Formict), y los datos primarios del cuestionario aplicado a coordinadores de NITs de tres estados del sur del país. Se ha utilizado análisis del contenido para la interpretación de los datos. Los resultados revelaron algunas dificultades en el proceso de institucionalización de NITs, ausencia de una cultura emprendedora, poca autonomía y obstáculos burocráticos en el reclutar de personal, demostrado en el modelo de organización del campo, basado en el pilar regulativo institucional. La conclusión es que los 
NITs están en fase de habitualización, la etapa de sedimentación y el proceso de institucionalización varían de acuerdo con la institución, aunque el mimetismo ha sido observado en sus acciones.

Palabras-clave: La Innovación. Sistema Nacional de Innovación. Núcleos de Innovación Tecnológica. Transferencia de Tecnología.

\section{INTRODUÇÃO}

Os Sistemas Nacionais de Ciência, Tecnologia e Inovação (CT\&I) visam soluções que resultem em melhorias econômicas, tecnológicas e inovação (LOTUFO, 2009). A inovação é produzida em interações entre diferentes instituições, muitas vezes no âmbito da tríplice hélice (ETKOWITZ; LEYDESDORFF, 2000). No contexto brasileiro, a Lei de Inovação, promulgada em 2004, estabeleceu a obrigatoriedade para universidades e institutos públicos de pesquisa e tecnologia estruturarem um órgão, constituído por uma ou mais Instituições de Ciência e Tecnologia (ICTs), voltado à gestão da política de inovação, que passou a ser denominado Núcleo de Inovação Tecnológica (NIT) (BRASIL, 2004). Posteriormente, em 2016, foi aprovado o novo marco legal da inovação, conhecido como Código de Ciência, Tecnologia e Inovação (Lei $\mathrm{n}^{\circ}$ 13.243/2016), atribuindo maior autonomia às ICTs, aos pesquisadores e, principalmente, aos NITs.

A estruturação dos NITs foi promulgada pela Lei de Inovação ${ }^{\circ}$ 10.973/2004, que dispõe sobre incentivos à inovação e à pesquisa científica e tecnológica no ambiente produtivo. A referida lei atribuiu às ICTs a função de estruturar um órgão interno, denominado Núcleo de Inovação Tecnológica (NIT), com a função de gerir as políticas institucionais de inovação, com as seguintes competências mínimas: (i) zelar pela manutenção da política institucional de estímulo à proteção das criações, licenciamento, inovação e outras formas de transferência de tecnologia; (ii) avaliar e classificar os resultados decorrentes de atividades e projetos de pesquisa; (iii) avaliar solicitação de inventor independente para adoção de invenção; (iv) opinar pela conveniência e promover a proteção das criações desenvolvidas na instituição; (v) opinar quanto à conveniência de divulgação das criações desenvolvidas na instituição passíveis de proteção intelectual; (vi) acompanhar o processamento dos pedidos e a manutenção dos títulos de propriedade intelectual da Instituição, foram acrescidas as seguintes competências; (vii) desenvolver estudos de prospecção tecnológica e de inteligência competitiva no campo da propriedade intelectual, de forma a orientar as ações de inovação da ICT; (viii) desenvolver estudos e estratégias para a transferência de inovação gerada pela ICT; (ix) promover e acompanhar o relacionamento da 
ICT com empresas; e (x) negociar e gerir os acordos de transferência de tecnologia oriunda da ICT.

Contudo, apesar de ter estabelecido mecanismos de estímulo ao engajamento de entes públicos em atividades de inovação com empresas, a Lei de Inovação não foi suficiente para traduzir-se em um efetivo instrumento promotor da interação público-privada para a realização da atividade inovativa no Brasil (RAUEN, 2016). A despeito do fato de que desde 2004 há um preceito legal que estabelece a criação de NITs, a implementação da maioria desses Núcleos nas ICTs ocorreu, predominantemente, a partir de 2008, sem clareza sobre formas de institucionalização, na medida em que sua atuação precisa propiciar um ambiente favorável à transferência de tecnologia e à proteção do conhecimento, atuando como interlocutores com o setor privado.

Por sua vez, a institucionalização é uma abordagem que procura explicar como e porque estruturas e processos tornam-se legitimados (DIMAGGIO; POWELL, 2005; VORONOV; DE CLERCQ; HININGS, 2013; SHERER; PALAZZO; SEIDL, 2013; MIRABEAU; MIGUIRE, 2014; CHANDLER; HWANG, 2015). Stevens, Xie e Peng (2015) consideram pertinente mencionar uma visão baseada em legitimidade, no campo dos estudos de estratégia a partir da perspectiva institucional. Para Zucker (1977), o grau de institucionalização é indicado pela conformidade dos indivíduos ao comportamento de outros. A habitualização representa a formalização de arranjos em políticas e procedimentos, processos que resultam em estruturas e que podem ser classificadas como um estágio de préinstitucionalização. A ação de objetivação constitui-se em fator de institucionalização e representa um dos componentes chave do processo de institucionalização. Outro componente é a sedimentação, que se apoia na continuidade histórica da estrutura. A sedimentação é um estágio de institucionalização total, relacionada com a perpetuação da estrutura por longo período (TOLBERT; ZUCKER, 1998).

Nesse sentido, esta pesquisa teve como objetivo conhecer como ocorreu a institucionalização de NITs, implantados no sul do Brasil, considerando que estes representam importante pilar no SNCT\&I. A fim de atingir o objetivo proposto, inicialmente apresenta-se uma revisão teórica sucinta sobre institucionalização e processo institucional e, em seguida, são descritos os procedimentos metodológicos, seguidos da apresentação e análise dos dados da pesquisa.

\section{CONSIDERAÇÕES SOBRE INSTITUCIONALIZAÇÃO E PROCESSO INSTITUCIONAL}


A perspectiva institucional é uma abordagem que procura entender, dentre outros aspectos, como e porque estruturas e processos tornam-se legítimos (DIMAGGIO; POWELL, 2005; TOLBERT; DAVI; SINE, 2011), isto é, prática, moral e cognitivamente aceitáveis ou, mesmo, recomendáveis do ponto de vista de algum grupo ou em sentido amplo (STEVENS; XIE; PENG, 2015). Trata-se de um processo social de ordenação, produzido e reproduzido em um dado sistema social (MACHADO-DA-SILVA; FONSECA; CRUBELLATE, 2005; QUATTRONE, 2015), sendo associado à compreensão de como ocorrem diversos tipos de respostas possíveis a pressões socialmente difundidas que tentam definir o modo de agir de atores sociais e organizações (GUARIDO FILHO, 2008; FUENTELSAZ; GARRIDO; MAICAS, 2015; SAKA-HELMHOUT; DEEG; GREENWOOD, 2016; BERRONE; FOSFURI; GELABERT; GOMEZ-MEJIA, 2013; FAULCONBRIDGE; MUZIO, 2016; VORONOV; DE CLERCQ; HININGS, 2013; GREENWOOD et al., 2011).

Contudo, deve-se reconhecer a dinamicidade desse processo, "sempre em construção" (CRUBELLATE, 2007, p. 215) e observado em níveis variados, sendo que alguns padrões de comportamento social estão mais sujeitos que outros à modificação e, até mesmo, à eliminação. Tais padrões comportamentais podem mudar em relação ao grau em que estão imbricados no sistema social e, assim, podem variar em termos de sua estabilidade e de seu poder em determinar comportamentos (TOLBERT; ZUCKER, 1998; TOLBERT et al., 2011).

Ao lado desse aspecto, deve-se reconhecer a dinâmica de respostas produzidas por atores sociais - indivíduos e organizações - às pressões de institucionalização, que envolvem uma série de aspectos, desde a natureza da pressão institucional (se coercitiva, por exemplo), o grau de complexidade institucional do ambiente, o grau de compromisso de stakeholders importantes para com as pressões institucionais, valores e interpretação predominantes nos grupos e atores organizacionais relevantes, práticas, dentre muitos outros elementos. Todos esses aspectos interferem, mais ou menos diretamente, na dinâmica de relação entre pressões institucionais e respostas organizacionais (GREENWOOD et al., 2011; RAAIJMAKERS et al., 2015; SMETS; MORRIS; GREENWOOD, 2012; CHANDLER; HWANG, 2015; BINDER, 2007; SAKA-HELMHOUT et al., 2016).

A institucionalização pode ser compreendida por meio de um conjunto de componentes- ou etapas-chaves correspondentes à habitualização, objetivação e sedimentação, permeadas ou mediadas, elas próprias, por uma série de atributos vinculados às próprias organizações e seus atores sociais no exercício de sua capacidade de agência ou no simples esforço para elaboração de respostas estratégicas às pressões originadas daquele processo de institucionalização. Assim, a habitualização envolve a geração de "novos 
arranjos estruturais em resposta a problemas ou conjuntos de problemas específicos, como também a formalização de tais arranjos em políticas e procedimentos" (BERGER; LUCKMANN, 1998, p.78). Nesse caso, as estruturas podem ser classificadas como em um estágio de pré-institucionalização (TOLBERT; ZUCKER, 1998). Sua própria conformação pode ser produto do exercício de empreendedorismo institucional (BATTILANA; LECA, 2009; COLYVAS; POWELL, 2009; DORADO, 2013).

Outro estágio, o de objetivação, implica em certo grau de consenso social a respeito do valor da estrutura e a crescente adoção pelas instituições com base nesse consenso (TOLBERT; ZUCKER, 1998). Esse estágio corresponde à semi-institucionalização, na qual é mais provável surgir e tornar-se relevante certa complexidade institucional (que implica multiplicidade e até contradição de pressões institucionais), resultando em elevado esforço para elaboração e legitimação de respostas adequadas ao ambiente ou mesmo a acomodação daquelas várias pressões (OLIVER, 1991; GREENWOOD et al., 2011; RAAIJMAKERS et al., 2015; BINDER, 2007). Para além do exercício intencional de elaboração de respostas estratégicas, os próprios atributos organizacionais e, mesmo, fatores de contingência são significativos - ao lado das pressões institucionais - na conformação das estruturas e processos (GREENWOOD; HININGS; WHETTEN, 2014).

A sedimentação, por fim, se apoia na continuidade histórica da estrutura e, especialmente, em sua sobrevivência pelas várias gerações de membros da organização (TOLBERT; ZUCKER, 1998). Nesse nível de institucionalização pode ocorrer o que Colyvas e Powell (2009, p. 88) consideram como autorreforço do padrão ou estrutura institucionalizada. Discutindo especificamente o contexto universitário e tecnológico norteamericano, Colyvas e Powell (2009) distinguem três períodos de desenvolvimento da relação entre universidades e empresas em torno da transferência de tecnologia: um inicial, de iniciativas localizadas e altamente idiossincráticas; um intermediário, que surge a partir de uma legislação específica e no qual a expansão da atividade de transferência tecnológica é fortemente dependente do suporte estatal; e, finalmente, um terceiro, no qual tais práticas se tornam altamente difundidas e aceitas no âmbito das universidades. Para eles, esta difusão e aceitação correspondem, exatamente, ao processo de institucionalização.

Mesmo face a padrões altamente difundidos e aceitos, entretanto, a relação entre organizações e ambiente pode conservar espaço para diversificação de ações e respostas, com base, por exemplo, no que vem sendo chamado de ingenuidade organizacional, isto é, a capacidade de criar e implementar alternativas sob restrições estruturais (WALKER; SCHLOSSER; DEEPHOUSE, 2014), ou mesmo na capacidade de empreendedorismo 
institucional (HENFRIDSSON; YOO, 2014).

De modo geral, as instituições - no que concerne ao seu conteúdo -contribuem para a redução da incerteza e para minimizar o risco do comportamento individual. Elas definem os papéis dos atores, como também o comportamento esperado deles em dadas estruturas. Em contrapartida, elas oferecem estabilidade. De acordo com Scott (2001), a base da legitimação institucional pode ser compreendida em três pilares: o regulativo, o normativo e o cognitivo (ver também BERRONE et al., 2013; ANG; BENISCHKE; DOH, 2015), como mostrado no Quadro 1.

Quadro 1 - Os três pilares segundo Scott (2001)

\begin{tabular}{|l|l|l|l|}
\hline \multirow{2}{*}{ Elementos } & \multicolumn{3}{|c|}{ Pilares } \\
\cline { 2 - 4 } & Regulativo & Normativo & Cultural-Cognitivo \\
\hline \multirow{2}{*}{ Bases de Conformidade } & Utilidade & Obrigação social & $\begin{array}{l}\text { Aceitação de pressupostos e } \\
\text { entendimento compartilhado }\end{array}$ \\
\hline Bases de Ordem & Regras Regulativas & Expectativas da adesão & Esquemas Constitutivos \\
\hline Mecanismos & Coercitivo & Normativo & Mimético \\
\hline Lógica & Instrumentalidade & Adequação & Ortodoxa \\
\hline Indicadores & Regras, leis e sanções & Certificado e Aceitação & $\begin{array}{l}\text { Crença comum e lógica } \\
\text { compartilhada de ação }\end{array}$ \\
\hline Base de Legitimidade & $\begin{array}{l}\text { Legalmente } \\
\text { sancionado }\end{array}$ & Moralmente Governado & $\begin{array}{l}\text { Conceitualmente correto e } \\
\text { culturalmente sustentado }\end{array}$ \\
\hline
\end{tabular}

Fonte: Scott (2001, p. 52).

Os elementos de caráter regulador caracterizam-se pela ênfase na fixação de normas e nas ações de sanção e coerção. A lógica da instrumentalidade contribui para a fixação dos mecanismos coercitivos, bem como para legitimidade das leis, regras e sanções estabelecidas.

O pilar normativo reflete-se no modo como as estruturas assumidas pelas organizações são derivadas da pressão exercida pelas normas e valores. Os valores representam concepções do preferível ou do desejado e as normas especificam como deveriam ser realizadas as tarefas. Nesse sentido, os elementos normativos abrangem representações coletivas da realidade e podem ser observados em diferentes níveis: no nível da sociedade, no qual normas e valores determinam comportamentos apropriados, no nível dos setores, nos quais instituições determinam modos e códigos de conduta, e no nível das comunidades.

O pilar cognitivo enfatiza representações internas e do ambiente, sendo que a escolha dos atores é limitada pelos modos como o conhecimento é construído (SCOTT, 2001). Esse pilar propõe que sejam valorizadas também as interpretações subjetivas das ações, somando as representações que os indivíduos fazem de seus ambientes configuradores de suas ações. 
Scott (2001) observa que os diferentes pilares devem ser vistos como alternativas analíticas, compreendendo diferentes facetas de um mesmo fenômeno. A separação em pilares não deve ser reificada, de modo a se rejeitar sua interdependência e complementaridade. Também no que concerne aos pilares, a recente análise institucional baseia-se na relação mútua entre ambiente e organizações para entender o processo de desenvolvimento institucional e seu impacto sobre aquelas organizações (BATTILANA; LECA, 2009; CHANDLER; HWANG, 2015).

Deste modo, os elementos institucionais cognitivos são menos sujeitos, em geral, à contestação, uma vez que configuram os mapas cognitivos de gestores e stakeholders relevantes, resultando em limitação do escopo de soluções admitidas como possíveis ou aceitáveis (BERRONE et al., 2013; ANG; BENISCHKE; DOH, 2015). Já o pilar regulativo tende a gerar maior mimetismo entre organizações, em busca de soluções para contextos específicos (ANG; BENISCHKE; DOH, 2015). Ainda de acordo com os mesmos autores, o mimetismo é um dos principais resultados quando ocorrem fortes pressões do pilar normativo, mesmo porque, em geral, o ambiente regulativo tende a refletir aspectos do ambiente normativo (HOLMES et al., 2013; ANG; BENISCHKE; DOH, 2015). Berrone et al. (2013), entretanto, identificaram (no que concerne a inovações em termos de gestão ambiental) que pressões institucionais - de ordem regulativa e também normativa - tendem a produzir, sob certas condições, maior impulso para inovação e maior heterogeneidade organizacional (ver, também, HAUNSCHILD; CHANDLER, 2008).

\section{PROCEDIMENTOS METODOLÓGICOS}

Para compreender o processo de institucionalização de NITs do sul do Brasil foi realizada uma pesquisa qualitativa e exploratória, esta última justificada quando há pouco conhecimento sobre o fenômeno (COOPER; SCHINDLER, 2003).

Como fontes de dados, foram coletados dados secundários e primários. Os secundários foram extraídos dos relatórios do Formulário para Informações sobre a Política de Propriedade Intelectual das ICTs (Formict) brasileiras, cujo preenchimento é coordenado pelo Ministério da Ciência, Tecnologia e Inovação (MCTI), referente aos anos de 2011, 2012, 2013, 2014 e 2015, que contêm dados sobre a implantação e o desenvolvimento dos Núcleos em ICTs públicas e privadas.

Os dados primários foram obtidos por meio de um questionário estruturado, com nove questões abertas destinadas a conhecer o processo de institucionalização dos NITs, versando 
sobre o papel dos participantes da pesquisa na implantação do Núcleo, bem como sobre a descrição da implementação do NIT na ICT, enfatizando dificuldades, resistências e atores envolvidos. Além disso, o questionário abrangeu questões sobre regulamentações internas e sobre contatos com outros Núcleos.

Os participantes da pesquisa foram identificados entre os associados ao Fórum Nacional de Gestores de Inovação e Transferência de Tecnologia (Fortec) do Sul do país. O Fortec foi constituído em 2006 e tem como principais objetivos disseminar a cultura da inovação, da propriedade intelectual e da transferência de tecnologia; auxiliar na criação e institucionalização dos NITs, e de outras instâncias gestoras de inovação; apoiar os NITs em suas gestões junto ao poder público e demais organizações da sociedade civil; promover a cooperação e atuação em rede entre seus associados; contribuir para a proposição de políticas públicas relacionadas à sua área de atuação; incentivar a pesquisa, o desenvolvimento científico e tecnológico, a inovação, a propriedade intelectual e a transferência de tecnologia no âmbito nacional, estadual e municipal; bem como promover a cooperação e o intercâmbio com Associações e entidades públicas ou privadas nacionais e internacionais (FORTEC, 2015).

Foram enviados questionários aos 29 coordenadores de NITs em ICTs dos estados do Sul (Rio Grande do Sul, Santa Catarina e Paraná), participantes do Fortec, dos quais, dez coordenadores devolveram o questionário preenchido. Os Núcleos que participaram da pesquisa foram: 3 de ICTs do Paraná, 2 de Santa Catarina e 5 do Rio Grande do Sul. No Paraná, todos eram de universidades públicas estaduais. Em Santa Catarina, um de ICT pública federal e um de fundação de direito privado. No Rio Grande do Sul, um era Centro Universitário, um de universidade confessional, dois de fundações de direito privado e um de ICT federal.

Antes de enviar os questionários os Núcleos foram contatados por telefone, explicando-se os objetivos da pesquisa e assegurando o anonimato e a livre adesão a pesquisa. Posteriormente, foram enviados os questionários em anexo a correspondências eletrônicas para preenchimento pelos coordenadores dos Núcleos.

Para análise dos dados secundários foi utilizada estatística descritiva, comparando-se os percentuais entre os anos analisados, apresentados em forma de tabelas. Para os dados primários, foram empregadas técnicas usuais da análise de conteúdo para decifrar, em cada texto, o núcleo emergente que servisse ao propósito da pesquisa, ou seja, para identificar aspectos associados à institucionalização dos referidos NITs. Essa etapa consistiu em um processo de codificação e interpretação das informações contidas nos documentos coletados e 
nos questionários (BAUER; GASKELL, 2007). Por meio de análise interpretativa buscou-se identificar elementos explicativos da estruturação e legitimação dos referidos núcleos. $\mathrm{O}$ material analisado foi codificado nas categorias explicativas da institucionalização: origem dos NITs, bases da institucionalização e dificuldades de institucionalização. Na categoria bases de institucionalização foram inseridos os pilares normativos, regulativos e cognitivos.

Algumas limitações podem ser mencionadas, como a dos dados secundários serem apenas nacionais, pois o Formict não apresenta dados por região. Quanto aos dados primários, a limitação da pesquisa foi contar com a participação apenas dos coordenadores dos NITs.

\section{APRESENTAÇÃO E ANÁLISE DOS RESULTADOS}

Inicialmente, para fins de contextualização, são apresentadas algumas considerações sobre a origem dos NITs.

\subsection{A ORIGEM DOS NITS}

Embora a Lei de Inovação tenha contribuído para a disseminação dos NITs no Brasil, Torkomian (2009) esclarece que dez anos antes da promulgação dessa Lei já existiam em muitas universidades estruturas análogas aos NITs, contudo, com diferentes denominações, como escritório de transferência de tecnologia, núcleo de propriedade intelectual, agência de inovação, dentre outras. Lobato et al. (2000) destacam que alguns institutos de pesquisas e universidades foram pioneiros em regulamentar o processo de inovação, como a Universidade Federal de Minas Gerais em 1977, a Universidade de São Paulo e a Universidade Estadual de Campinas em 1989, a Empresa Brasileira de Pesquisa Agropecuária e a Fundação Oswaldo Cruz em 1996. A Universidade de Campinas institucionalizou seu núcleo de gestão tecnológica em 1989, bem como o Escritório de Transferência de Tecnologia (ETT), sendo que nove anos mais tarde, em 1998, esse Escritório foi sucedido pelo Escritório de Difusão de Tecnologia (Edistec). Em 2003, antes mesmo da aprovação da Lei de Inovação, foi criada a Agência de Inovação Inova Unicamp, com o objetivo de atuar com uma nova amplitude no processo de inovação, sucedendo assim o Edistec (TOLEDO, 2009).

A Lei de Inovação alavancou a criação dos NITs, mas estes se encontram em estágios de estruturação e níveis de maturidade diferenciados. Com isso, a data de criação dos NITs não reflete necessariamente o início das estratégias das ICTs com o tema inovação, como relatam Pires e Quintella (2015), podendo derivar de um processo de desenvolvimento e 
amadurecimento institucional ou unicamente em função do atendimento ao disposto na lei.

Estudo realizado por Dias (2010) junto a 157 NITs brasileiros revelou que a distribuição dos anos de implantação desses núcleos apresenta-se entre 1960 e 2009, mas, de forma geral, eles concentraram-se no ano de 2004. Entre os NITs participantes desta pesquisa, um foi implementado em 2005 e outro em 2011, sendo o restante em 2006.

\subsection{BASES DA INSTITUCIONALIZAÇÃO}

A situação atual dos NITs no Brasil tem seu registro no panorama gerado pelos dados coletados pelo MCTI, por meio do Formict. Em 2014 esse formulário foi respondido por 264 Instituições, sendo 194 públicas e 70 privadas. A pesquisa revelou ainda que existia no país 180 instituições com NITs implementados (68,2\%), 54 em fase de implementação $(20,5 \%)$ e 30 ainda implementados (11,4\%) (MCTI, 2015).

É possível perceber o crescimento anual da implementação de NITs no país, na medida em que foram implementados 94 no ano base de 2010; 116 em 2011; 141 em 2012; 166 em 2013; e 180 em 2014. Observa-se que o incremento ocorreu em instituições de natureza privada, passando de 67 em 2013 para 70 em 2014. Os dados dos relatórios mostram que o número de NITs aumentou anualmente, predominantemente em instituições públicas e nas regiões sul e sudeste. Em termos de legitimação, as estruturas estavam formalizadas nos 180 NITs existentes no ano de 2014. Alcançar a totalidade da implementação dos NITs nas instituições públicas e consolidar aqueles já implementados consistem desafios na atualidade (MCTI, 2015).

No tocante à institucionalização dos núcleos, especificamente quanto à habitualização, esta se reflete nas atividades da política de inovação, ou seja, em documentos formais com diretrizes gerais que norteiam a atuação das instituições nas ações ligadas à inovação, à proteção intelectual e à transferência de tecnologia. De acordo com os relatórios Formict consultados, as ICTs que implementaram NITs desenvolveram diversas atividades relacionadas à política de inovação, cujos percentuais são apresentados na Tabela 1.

Em relação aos resultados apontados no relatório Formict do ano base 2014, verificase que a única atividade que apresentou redução em relação ao de 2013 foi a de ensino em temas associados à inovação, que passou de 79\% em 2013 para 77,3\% em 2014. Quanto à política voltada à confidencialidade, o percentual manteve-se constante, 92,3\%. 
Tabela 1 - Percentual das atividades da política de inovação implementadas nos NITs

\begin{tabular}{l|c|c|c|c|c}
\hline \multicolumn{1}{c|}{ Atividades/Ano base } & $\begin{array}{c}\mathbf{2 0 1 0} \\
(\boldsymbol{\%})\end{array}$ & $\begin{array}{c}\mathbf{2 0 1 1} \\
(\boldsymbol{\%})\end{array}$ & $\begin{array}{c}\mathbf{2 0 1 2} \\
(\boldsymbol{\%})\end{array}$ & $\begin{array}{c}\mathbf{2 0 1 3} \\
(\boldsymbol{\%})\end{array}$ & $\begin{array}{c}\mathbf{2 0 1 4} \\
(\boldsymbol{\%})\end{array}$ \\
\hline $\begin{array}{l}\text { Desenvolvimento de projetos de cooperação } \\
\text { com terceiros }\end{array}$ & 92 & 91,3 & 89,5 & 90,6 & 93,8 \\
\hline Confidencialidade & 87 & 91,3 & 95,5 & 92,3 & 92,3 \\
\hline Acordos de parcerias & 86 & 82,5 & 85,7 & 87,8 & 91,8 \\
\hline $\begin{array}{l}\text { Contrato prevendo a titularidade da propriedade } \\
\text { intelectual e a participação nos resultados }\end{array}$ & 69 & 74,6 & 76,7 & 78,5 & 84 \\
\hline Alianças estratégicas & 73 & 77,8 & 79,7 & 77,3 & 79,4 \\
\hline $\begin{array}{l}\text { Atividade de ensino em temas relacionados à } \\
\text { inovação }\end{array}$ & 73 & 74,6 & 78,9 & 79 & 77,3 \\
\hline Prestação de serviços & 68 & 72,2 & 73,7 & 75,7 & 75,8 \\
\hline $\begin{array}{l}\text { Compartilhamento de instalações e permissão } \\
\text { de utilização }\end{array}$ & 41 & 68,3 & 60,9 & 66,9 & 69,6 \\
\hline $\begin{array}{l}\text { Participção do pesquisador em resultados } \\
\text { econômicos }\end{array}$ & 58 & 62,7 & 60,2 & 58 & 65,5 \\
\hline Contratos de transferência e licenciamento & 54 & 61,1 & 66,2 & 60,2 & 64,9 \\
\hline Retribuição pecuniária aos pesquisadores & 50 & 53,2 & 54,1 & 49,2 & 56,7 \\
\hline Estímulo ao inventor independente & 45 & 50,8 & 54,9 & 51,4 & 55,2 \\
\hline Bolsa de estímulo à inovação & 56 & 54,8 & 56,4 & 53 & 54,6 \\
\hline $\begin{array}{l}\text { Cessão de direitos sobre a criação para que o } \\
\text { criador os exerça em seu nome }\end{array}$ & 35 & 41,3 & 43,6 & 42,5 & 46,4 \\
\hline $\begin{array}{l}\text { Afastamento para prestar colaboração a outra } \\
\text { ICT }\end{array}$ & 24 & 23 & 27,8 & 22,7 & 29,4 \\
\hline $\begin{array}{l}\text { Licenças sem remuneração para o pesquisador } \\
\text { constituir empresa }\end{array}$ & 18 & 18,3 & 19,5 & 18,8 & 23,2 \\
\hline Outras & 18 & 20,6 & 18 & 16 & 16,5 \\
\hline
\end{tabular}

Fonte: elaborado pelos autores a partir de Formict $(2015 ; 2014 ; 2013 ; 2012 ; 2011)$.

O relatório de 2014 revela que houve incremento em praticamente todas as atividades relacionadas à política de inovação em relação ao ano de 2013, sendo que as mais significativas referem-se à: retribuição pecuniária aos pesquisadores, passando de 49,2\% para $56,7 \%$; afastamento para prestar colaboração a outra ICT, de $22,7 \%$ para $29,4 \%$; contrato prevendo a titularidade da propriedade intelectual e a participação nos resultados, mudando de $78,5 \%$ para $84 \%$; participação do pesquisador em resultados econômicos, variando de $58 \%$ para 65,5\%; e licenças sem remuneração para o pesquisador constituir empresa, que passou de $18,8 \%$ para $23,2 \%$.

Do mesmo modo, observou-se ainda aumento quanto aos acordos de parcerias, passando de $87,8 \%$ para $91,8 \%$, como também quanto aos contratos de transferência e licenciamento, variando de 60,2\% para 64,9\%, e estímulo ao inventor independente, alterando de $51,4 \%$ para $55,2 \%$. Outras variações foram: cessão de direitos sobre a criação para que o criador os exerça em seu nome, elevando-se de 42,5\% para 46,4\%; desenvolvimento de projetos de cooperação com terceiros, de 90,6\% para 93,8\%; estabelecimento de alianças estratégicas, que mudou de 77,3\% para 79,4\%; compartilhamento de instalações e permissão 
de utilização, passando de 66,9\% para 69,6\%; bolsa de estímulo à inovação, que variou de $53 \%$ para 54,6\%; e prestação de serviços, com pequena variação de 75,7\% para 75,8\%.

Quanto aos resultados apontados pelo Formict nos anos de 2012 e 2013, observou-se que houve pequena redução na atividade confidencialidade (95,5\% para 92,3\%). Outra redução observada foi na participação do pesquisador em resultados econômicos, pois em 2012 era de 60,2\% e em 2013 caiu para 58\%. O mesmo ocorreu em relação às bolsas de estímulo à inovação, que reduziram de 56,4\% para 53\%, assim como o estímulo ao inventor independente, que passou de $54,9 \%$ para $51,4 \%$, e alianças estratégicas, que alterou de $79,7 \%$ para $77,3 \%$. Redução significativa foi observada quanto à retribuição pecuniária aos pesquisadores, que passou de $54,1 \%$ para $49,2 \%$, mas passando para $56,7 \%$ em 2014 ; bem como em relação ao afastamento para prestar colaboração a outra ICT $(27,8 \%$ para $22,7 \%)$ e à celebração de contrato de transferência e licenciamento (66,2\% para 60,2\%). Nota-se que apesar do aumento no número de ICTs públicas que implementaram políticas de inovação nos anos de 2012 e 2013 (68,9\% para 69,4\% das ICTs), observou-se redução em atividades associadas a pesquisadores.

A habitualização para os NITs, conforme mostram os dados do Quadro 2, é principalmente alicerçada em três atividades: desenvolvimento de projetos de cooperação com terceiros, garantia de confidencialidade e acordos de parcerias. A atividade menos relevante é a licença sem remuneração para pesquisador constituir empresa.

Quanto ao pilar regulativo, a institucionalização dos NITs foi decorrente de um processo indutivo em relação a projetos e a objetivos comuns, sendo sustentada pela legitimidade de seus elementos constituintes, ou seja, pelos 'scripts' comportamentais específicos de ações, tais como as descritas anteriormente. De acordo com o pilar regulativo (SCOTT, 2001), as bases de conformidade dos NITs foram estabelecidas pelas leis, como pode ser observado no Quadro 2.

Algumas ICTs desenvolveram, implementaram e institucionalizaram suas políticas de pesquisa e seus órgãos de transferência de tecnologia antes mesmo da Lei de Inovação, mas elas foram exceções, pois o processo que deflagrou a institucionalização quase generalizada foi derivado da Lei de Inovação e outras correlatas, constituindo-se o pilar regulativo, como mostrado no Quadro 2. 
Quadro 2 - Análise tendo o pilar regulativo como referência

\begin{tabular}{|c|c|c|}
\hline Bases de conformidade & Bases de ordem & Mecanismos \\
\hline $\begin{array}{l}\text { Lei Federal de Inovação: os NITs } \\
\text { têm a finalidade de gerir a } \\
\text { política de inovação das ICTs. } \\
\text { Lei de Propriedade Industrial, } \\
\text { Resoluções e Instruções } \\
\text { Normativas do Instituto Nacional } \\
\text { da Propriedade Industrial } \\
\text { voltados para questões } \\
\text { relacionadas à Propriedade } \\
\text { Intelectual. } \\
\text { Lei de Direitos Autorais } \\
\text { Lei de Programa de Computador } \\
\text { Leis Estaduais de Inovação, que } \\
\text { dispõem sobre medidas de } \\
\text { incentivo à inovação e à pesquisa } \\
\text { científica e tecnológica em } \\
\text { ambiente produtivo nos } \\
\text { Estadosda Federação. } \\
\text { Decretos Estaduais a que } \\
\text { regulamentam as medidas de } \\
\text { incentivo à inovação e à pesquisa } \\
\text { científica e tecnológica } \\
\text { Lei do Bem no } 11.196 / 2005, \text { que } \\
\text { estabelece os incentivos e } \\
\text { benefícios fiscais. }\end{array}$ & $\begin{array}{l}\text { Institucionalização nas estruturas } \\
\text { das IES: das } 264 \text { instituições que } \\
\text { preencheram o Formict do ano base } \\
\text { de } 2014 \text { e, consequentemente, } \\
\text { possuem NITs, } 194 \text { apresentaram-se } \\
\text { como instituições públicas (74,49\%) e } \\
70 \text { como privadas }(26,52 \%) \text {. No que } \\
\text { diz respeito à natureza das } \\
\text { instituições, 69,1\% correspondem ao } \\
\text { nível federal, 27,8\% estadual e 3,1\% } \\
\text { municipal. } \\
\text { Relatórios que deem ser } \\
\text { preenchidos junto ao MCTI: } \\
\text { Formulário para Informações sobre a } \\
\text { Política de Propriedade Intelectual } \\
\text { das Instituições Científicas e } \\
\text { Tecnológicas do Brasil (Formict). O } \\
\text { formulário deve ser preenchido } \\
\text { anualmente pelas ICTs por } \\
\text { determinação da Lei de Inovação } \\
\text { (nº.10.973, de 02.12.2004), que } \\
\text { estabelece, no Artigo 17, que as ICTs } \\
\text { deverão enviar ao MCTI informações } \\
\text { sobre sua política de propriedade } \\
\text { intelectual, criações desenvolvidas, } \\
\text { proteções requeridas e concedidas e } \\
\text { contratos de licenciamento ou } \\
\text { transferência de tecnologia firmados. }\end{array}$ & $\begin{array}{l}\text { Coercitivo. } \\
\text { Não há punição prevista em lei, } \\
\text { contudo a inexistência de NIT } \\
\text { pode inviabilizar a captação de } \\
\text { recursos federais e estaduais pelo } \\
\text { não atendimento de exigências } \\
\text { previstas em Edital. }\end{array}$ \\
\hline
\end{tabular}

Fonte: elaborado pelos autores.

De acordo com os dados primários desta pesquisa, legislações internas foram implementadas nas ICTs. Todas criaram um regulamento interno para normatizar a atuação dos NITs. Além disso, resoluções de Propriedade Intelectual e de Transferência de Tecnologia foram normativas instituídas em oito ICTs. Dois NITs formularam Regimentos para criação ou atuação de Agência de Inovação. Os NITs atuaram como indutores nas ICTs de Política de Pesquisa, Desenvolvimento e Inovação, bem como Política de Propriedade Intelectual. Um dos NITs implantou termos de sigilo, termos de contratos de transferência de tecnologia e de fluxos de trabalho. Outro criou uma resolução para empresas júniores e outra para atuação de incubadora de empresas. Quatro NITs contam com estrutura conjunta com incubadoras.

Para Scott (2001) as bases de legitimação não se restringem ao pilar regulativo, mas estendem-se aos pilares normativos e cognitivos. O Quadro 3 apresenta essas dimensões para os NITs, de acordo com dados primários e secundários da pesquisa. 
Quadro 3 - Análise tendo o pilar normativo como referência

\begin{tabular}{|c|c|c|}
\hline Obrigação Social & Expectativa de adesão & Normativo \\
\hline $\begin{array}{l}\text { Competências mínimas de um NIT } \\
\text { (Artigo } 16 \text { da Lei no } 10.973 / 2004) \text { : } \\
\text { I - Zelar pela manutenção da política } \\
\text { institucional de estímulo à proteção } \\
\text { das criações, licenciamento, inovação } \\
\text { e outras formas de transferência de } \\
\text { tecnologia; } \\
\text { II - Avaliar e classificar os resultados } \\
\text { decorrentes de atividades e projetos } \\
\text { de pesquisa; } \\
\text { III - Avaliar solicitação de inventor } \\
\text { independente para adoção de } \\
\text { invenção; } \\
\text { IV - Opinar pela conveniência e } \\
\text { promover a proteção das criações } \\
\text { desenvolvidas na instituição; } \\
\text { V - Opinar quanto à conveniência de } \\
\text { divulgação criações } \\
\text { desenvolvidas nas instituição, } \\
\text { passíveis de proteção intelectual; } \\
\text { VI - Acompanhar o processamento } \\
\text { dos pedidos e a manutenção dos } \\
\text { títulos de propriedade intelectual da } \\
\text { instituição. }\end{array}$ & $\begin{array}{l}\text { Conforme estabelecido pelo Art. } 16 \text { da } \\
\text { Lei de Inovação (Lei no } 10.973 / 2004 \text { ), } \\
\text { a ICT deverá dispor de NIT próprio ou } \\
\text { em associação com outras ICTs, com } \\
\text { a finalidade de gerir sua política de } \\
\text { inovação. Assim, de acordo com essa } \\
\text { Lei, todas as ICTs devem ter NITs } \\
\text { próprios ou compartilhados. }\end{array}$ & $\begin{array}{l}\text { Normas federais, estaduais e } \\
\text { das próprias ICTs. } \\
\text { Nas ICTs, segundo dados } \\
\text { desta pesquisa,foram criados } \\
\text { regimento interno para NITs, } \\
\text { regimento para agência de } \\
\text { inovação, termos de sigilo, } \\
\text { contratos de transferência de } \\
\text { tecnologia, fluxos de trabalho, } \\
\text { políticas de propriedade } \\
\text { intelectual e de pesquisa, } \\
\text { desenvolvimento e inovação. }\end{array}$ \\
\hline
\end{tabular}

Fonte: elaborado pelos autores.

O pilar normativo induz à adesão de códigos de conduta específicos. A obrigação social, que pode ser observada no Quadro 3, define os limites de atuação e o que se espera dos NITs. A expectativa de adesão é explícita na lei e visa criar legitimação, importante principalmente para os NITs iniciantes. Além destas, regulamentações formais como certificações e acreditações podem configurar o pilar normativo para reforçar o processo de institucionalização, embora elas ainda não tenham sido pensadas e implementadas. No entanto, não apenas mecanismos formais, mas também mecanismos informais podem reforçar o processo, como a confiança. Nesse sentido, a maioria dos NITs encontra-se ainda em processo de pré-institucionalização, conforme Zucker (1977), tendo já estabelecido o rol de atividades, encontrando-se, portanto, em fase de habitualização.

Quanto à obrigação social, os dados da pesquisa mostraram que alguns núcleos incorporaram outras ações, tais como: interação entre Universidade e Empresas, assessoria para captação de recursos para pesquisa, desenvolvimento e inovação (PD\&I) e coordenação de projetos de assessoria empresarial para indústrias. A realização de eventos para capacitação de empreendedores, eventos para estimular networks e palestras para estímulo à inovação foram mencionadas. Outra função citada por um dos núcleos foi a realização de eventos acadêmicos sobre inovação e propriedade intelectual, bem como premiação para projetos 
inovadores. Nesse sentido, apesar do mimetismo e de pressões institucionais na conformação das estruturas (GREENWOOD; HININGS; WHETTEN, 2014), o que muitas vezes deriva da forma como os Núcleos foram inseridos nas ICTs, nesta pesquisa foram também observadas ações caracterizando exercício de empreendedorismo institucional (BATTILANA; LECA, 2009; DORADO, 2013).

O pilar cultural cognitivo, de acordo com Scott (2001), completa o processo de institucionalização. Esse pilar está sujeito a variações ao longo do tempo, embora a tendência seja que este mude lentamente (SCOTT, 2001). Mecanismos miméticos asseguram a legitimação dos processos e introduzem a habitualização. No âmbito dos NITs, a habitualização encontra o desafio de promover a aproximação do meio empresarial e meio acadêmico.

As principais bases do pilar cognitivo são transmitidas em rede. Os NITs integram o Fortec, uma associação de representação dos responsáveis nas universidades, institutos de pesquisa e instituições gestoras de inovação pelo gerenciamento das políticas de inovação e das atividades relacionadas à propriedade intelectual e à transferência de tecnologia. $\mathrm{O}$ Quadro 4 resume o pilar cultural-cognitivo de acordo com Scott (2001).

Quadro 4 - Análise tendo o pilar cultural cognitivo como referência

\begin{tabular}{|l|l|}
\hline \multicolumn{1}{|c|}{ Pilar Cultural Cognitivo } & \multicolumn{1}{|c|}{ Mimético } \\
\hline $\begin{array}{l}\text { Principais pressupostos compartilhados: } \\
\text { Necessidade de sensibilização de áreas com potencial } \\
\text { de inovação e para tanto mantem contatos com }\end{array}$ & $\begin{array}{l}\text { Considerando o trabalho em rede, existe } \\
\text { compartilhamento de conhecimento entre os NITs. } \\
\text { SEBRAE, Associação Empresarial do Município e } \\
\text { FIEP. }\end{array}$ \\
$\begin{array}{l}\text { Necessidade de propiciar relacionamento entre as ICTs } \\
\text { e o setor empresarial. Bases de conhecimento são } \\
\text { buscadas em outras Agências de inovação, até mesmo de propriedade intelectual. } \\
\text { fora do próprio estado. }\end{array}$ & $\begin{array}{l}\text { Não há característica de concorrência. } \\
\text { Realidade de outros países serve para estimular, mas a } \\
\text { diferença entre eles inviabiliza cópia de modelos. }\end{array}$ \\
$\begin{array}{l}\text { Necessidade de promover a aproximação de de } \\
\text { pesquisadores com o setor empresarial. }\end{array}$ & \\
\hline
\end{tabular}

Fonte: elaborado pelos autores com base em Scott (2001) e dados da pesquisa.

A tendência ao mimetismo, de acordo com os dados da pesquisa, é ainda reforçada por outros contatos que os NITs mantêm, tendo sido constatado que dentro de cada Estado há uma interação entre os Núcleos. Outras conexões em rede mencionadas pelos participantes desta pesquisa foram: Serviço Brasileiro de Apoio às Micro e Pequenas Empresas (SEBRAE), Associação Empresarial do Município, Rede Estadual de Inovação, Rede Estadual de Propriedade intelectual, Secretaria Estadual de Tecnologia, Federação de Indústrias do Estado e Empresas locais co-titulares em pedidos de patentes e licenciadas. Um dos NITs mantém 
interação com a agência de inovação do Estado de São Paulo. Além disso, dois Núcleos mencionaram interação com a Financiadora de Estudos e Projetos (FINEP), por meio de financiamento de projetos de inovação, e com o Instituto Nacional de Propriedade Industrial (INPI).

As bases de legitimidade são asseguradas não apenas pelos instrumentos, como também pela legitimidade de Coordenadores, geralmente docentes com titulação de doutores. No âmbito das estruturas das ICTs, observou-se que em geral os Núcleos estão vinculados hierarquicamente ao Gabinete do Reitor ou às Pró-Reitorias de Pesquisa e Pós-Graduação. Pode supor que essa vinculação contribua para aumentar o grau de autonomia dos Núcleos, que é reduzido, como constataram Rodrigues e Gava (2016). Nesse sentido, a Lei n ${ }^{\circ}$ 13.243/2016, em seu artigo 16, acena com a possibilidade dos NITs serem juridicamente independentes.

Lotufo (2009) menciona que os NITs têm como atribuições: a) legais: funções de regulação e formalização, recebendo influência do departamento jurídico da ICT quanto ao depósito ou não de pedidos de patente e formalização de convênios com empresas, tendo o corpo funcional majoritariamente composto por advogados e especialistas em propriedade intelectual; b) administrativas: atuação do NIT como processo administrativo para a concretização de convênios e contratos ligados à interação ICT- empresa; e c) orientado a negócios: desenvolvimento de negócios a partir de resultados de pesquisas, sendo que o corpo funcional compreende as dinâmicas que permeiam a inovação, as pesquisas acadêmicas e o funcionamento do mercado. No entanto, o cumprimento dessas atribuições, que demonstraria a institucionalização dos núcleos, de acordo com dados desta pesquisa, apresenta algumas dificuldades que são comentadas a seguir.

\subsection{DIFICULDADES DE INSTITUCIONALIZAÇÃO}

Para Lotufo (2009) uma das dificuldades para a atuação efetiva de um NIT consiste no estabelecimento de políticas internas de cada instituição e de sua experiência no estabelecimento de convênios e contratos tecnológicos, pois de forma distinta ao que ocorre em relação aos convênios de pesquisa colaborativa, o contrato de licenciamento é de longa duração, envolve diversas fases de desenvolvimento, há risco na sua execução e demanda uma sólida segurança jurídica. Entre os NITs participantes desta pesquisa, dois contam com assessoramento externo para contratos e, nem mesmo os demais mencionaram essa dificuldade. 
A inconstância de políticas internas das universidades, constantemente alteradas com a troca de gestão, foi um dos aspectos mais enfatizados nesta pesquisa. Para alguns, o pioneirismo de instituições com atividades de ensino dificulta que priorizem a transferência de tecnologia ou a gestão da inovação e que incorporem uma política de ciência e tecnologia.

Outra dificuldade consiste na implementação de uma cultura empreendedora, voltada para a aceitação de integração entre universidade e setor produtivo. Este é um desafio desde a criação dos NITs, como relataram diversos participantes. Segundo eles, os NITs encontram dificuldades derivadas da compreensão do papel desses órgãos até mesmo no âmbito interno, não havendo clareza por parte da comunidade acadêmica e das empresas. O desafio da disseminação da cultura, segundo um dos participantes: "é sempre acompanhada de uma certa dose de desconfiança...convencer pesquisador a se tornar um empreendedor não é tarefa das mais fáceis".

A legitimação da infraestrutura foi outro aspecto, apontado pela maioria dos participantes, evidenciada na dificuldade em conquistar confiança interna no âmbito das ICTs, principalmente pela falta de apoio institucional, muitos casos apresentados desde a criação do NIT. Outro participante mencionou a dificuldade com a falta de visão administrativa sobre as necessidades do Núcleo e o engessamento do sistema universitário para contratação de pessoal. Nesse aspecto, foi mencionada como dificuldade a de encontrar recursos humanos habilitados para exercerem funções associadas a P D \&I.

Em síntese, os dados da pesquisa mostraram três grupos de dificuldades, comentadas anteriormente, associadas à instabilidade política interna das ICTs, à dificuldade em implementar uma cultura empreendedora e dificuldades estruturais, tais como pouca autonomia e dificuldades para contratação de pessoal. Diferentes resultados foram obtidos por Calzolaio, Correia e Dathein (2013), que identificaram o baixo dinamismo entre ICTs e empresas; a quase inexistência de financiamento privado para atividades de inovação e a insuficiente transferência de pesquisadores das ICTs para as empresas.

\subsection{ANÁLISE DO PROCESSO DE INSTITUCIONALIZAÇÃO}

O processo de institucionalização dos NITs derivou de um condutor institucional de natureza regulativa, qual seja, a promulgação da Lei de Inovação. A partir da categorização de situações proposta por Colyvas e Powell (2009), pode-se apontar a atual situação dos NITs e da transferência de tecnologia (Universidades-Empresas), no contexto empírico aqui investigado, como estando em estágio intermediário, sendo consequentemente dependente, 
em elevado grau, do suporte legal e político estatal. Isso significa, ao mesmo tempo, que a estrutura não está amplamente difundida e não é, ainda, intensamente assumida, como necessária ou desejada.

Tal constatação sugere prever certas consequências para a eficiência do sistema, já que seu funcionamento continua baseado na regulação e em pressão razoavelmente coercitiva. Em parte, ao menos, essa constatação explica dificuldades enfrentadas pelos NITs em sua institucionalização, conforme apontado ao final da seção anterior. Considerando-se a tradição formalista predominante no país (OLIVEIRA; MACHADO-DA-SILVA, 2001) tal estágio intermediário - com seus desdobramentos e entraves - pode representar risco para a construção de uma identidade empreendedora nas universidades brasileiras. Para enfrentar essa questão, a Lei de Inovação, no que tange aos NITs, passou por alterações com o novo marco legal de tecnologia, o que, entretanto, não é ainda garantia de que não se esteja reforçando o viés formalista, típico de nossa tradição nacional, como anteriormente apontado.

De qualquer modo, em janeiro de 2016, o novo marco legal de CT\&I foi aprovado, mediante a Lei $\mathrm{n}^{\circ} 13.243$, atribuindo maior liberdade e autonomia às ICTs, aos pesquisadores e principalmente aos NITs. De acordo com Rauen (2016), a nova lei avança em diversos pontos na promoção de um ambiente regulatório mais seguro e estimulante para a inovação no Brasil. No que tange aos NITs, a alteração de maior impacto foi a possibilidade de instituir personalidade jurídica própria, com possibilidade de maior autonomia para a realização de suas atividades e competências, tendo ainda a possibilidade de funcionar como uma fundação de apoio para projetos que resultem em novos serviços ou tecnologias. A nova Lei amplia o papel dos NITs, até então relacionado unicamente à gestão da propriedade intelectual e da transferência de tecnologias das ICTs. Apesar disso, Rodrigues e Gava (2016) ainda sustentam que a falta de autonomia dos Núcleos é um ponto crítico, que afeta negativamente a exigência de comportamento flexível para responder às demandas por inovação.

Os dados desta pesquisa mostraram, também, que a maioria dos núcleos encontra dificuldade para legitimação interna, isto é, no âmbito das ICTs. Até mesmo os representantes do NIT mais antigo participante desta pesquisa mencionaram a dificuldade com a "sensibilização de pesquisadores sobre a importância da propriedade intelectual”. Com isso, a institucionalização dos NITs precisa superar essas barreiras, a fim de avançar nos estágios de institucionalização nas IES, a fim de efetivamente cumprir o seu papel no sistema de inovação. A nova lei pressupõe que a possibilidade de instituir os NITs como órgãos independentes poderá solucionar essa questão. Entretanto, a questão de como será a institucionalização do NIT independente permanece aberta, considerando-se os aspectos do 
contexto formalista típico do ambiente da gestão pública e mesmo do contexto de gestão dos NITs. Se, por um lado, o contexto regulativo favorece as ações de natureza mimética, já que propiciam, por um lado, a difusão de respostas testadas e bem-sucedidas em relação ao marco legal, por outro, tendem a limitar de forma aguda a propensão à busca por soluções inusitadas e, consequentemente, a evolução do campo organizacional (SCOTT, 2001; COLYVAS, 2007).

Padrões de comportamento mimético contribuem, obviamente, para a institucionalização, já que fornecem respostas consideradas aceitáveis no contexto de operação ou no campo organizacional. Porém, elas tendem a limitar a possibilidade de inovação. É possível, entretanto, que o mimetismo ocorra simultaneamente a um processo de incorporação de elementos que favoreçam um comportamento adaptado aos contextos e aos recursos locais (ver, por exemplo, ANG; BENISCHKE; DOH, 2001; BINDER, 2007; WALKER; SCHLOSSER; DEEPHOUSE, 2014). Nesta pesquisa observou-se alguma iniciativa nesse sentido, caracterizando-se algum nível de empreendedorismo institucional, muito aquém, ainda, do que parece possível - ou é necessário - para o efetivo desenvolvimento da inovação nas IES.

Nesse processo de institucionalização, legitimidade e poder são elementos centrais. Zucker (1987) e Machado-da-Silva, Fonseca e Crubellate (2005) consideram que a legitimidade não é atributo objetivo ou dado, senão o resultado do próprio processo de institucionalização das estruturas. Com isso, ela decorre, em parte ao menos, do próprio trabalho institucional dos atores sociais que, posteriormente, tornam-se legítimos mediante práticas que disseminam aquelas estruturas que vão se tornando institucionalizadas. Nesse estudo, é importante salientar o papel dos coordenadores dos NITs como atores a sedimentarem o processo de institucionalização dos referidos Núcleos. Isso, entretanto, parece exigir a superação do estágio intermediário, em direção a um estágio de maior legitimação que, ao mesmo tempo, implique em espaços para agência e trabalho institucional daqueles atores com poder suficiente, e suporte institucional suficientemente claro, para elaborar respostas locais às necessidades de interação entre os Núcleos, o setor produtivo e a própria sociedade mais ampla (COLYVAS, 2007).

Um dos mais severos limites impostos pela permanência do modelo em seu estágio intermediário (isto é, altamente dependente do Estado e fortemente baseado em um pilar regulativo) é a dificuldade para a recombinação e harmonização de modelos de transferência de tecnologia (COLYVAS, 2007), sem o que determinadas versões tradicionais acabam por vincular excessivamente as práticas a uma lógica burocrática e ao poder que dela emana e 
que, frequentemente, está mais vinculada às atividades-meio das ICTs. Os dados desta pesquisa mostraram que os NITs não estão sedimentados, ao menos não em níveis elevados. A habitualização está amplamente alicerçada em regulamentos internos. Os dados mostraram também que não há objetivação e consenso social em torno da estrutura (TOLBERT; ZUCKER,1998). Segue-se que a difusão do modelo dos NITs não corresponde, ainda, à institucionalização da relação entre universidades e sociedade em torno da questão da inovação, que pode ser considerado uma das atividades-fim dos NITs e, provavelmente, das próprias IES.

Em termos de uma síntese final, aponta-se que a configuração do pilar regulativo (SCOTT, 2001) se dá, no caso dos NITs, com base em diversas leis e regulamentos no âmbito nacional, estadual e mesmo interno às ICSts. Já quanto ao pilar normativo, pode-se apontar que há adequação das estruturas para abrigar os NITs. Esses são considerados obrigatórios nas IES (o que nos remete, novamente, ao pilar regulativo), mas sua aceitação não é ampla, haja vista o comportamento dos pesquisadores, como apontaram os dados desta pesquisa. Quanto ao pilar cultural-cognitivo, apontou-se que ainda há pouco entendimento compartilhado e evidencia-se a ausência de uma lógica compartilhada de ação no que concerne à questão da inovação e da relação entre universidades e sociedade.

Evidencia-se, no âmbito das ICTs, a falta de discussão a respeito do papel dos atores envolvidos no processo da inovação. A complexidade institucional inerente ao processo de inovação (envolvendo lógicas de ordem administrativo-burocrática, científica, estatal, de mercado) exige dos sistemas a capacidade de assegurar compromisso dos vários stakeholders. A dinâmica de institucionalização, com sua dependência para com os processos de legitimação e compartilhamento de poder, não pode ser efetivamente conduzida por uma via apenas regulativa, especialmente porque algumas daquelas lógicas - e dos atores a elas vinculados, como é o caso da lógica científica e dos pesquisadores- não podem ser tratadas apenas por uma via legal, ou coercitiva. Esse aspecto é, do ponto de vista dos dados desta pesquisa, um dos principais entraves ainda presentes para a institucionalização dos NITs e do processo de inovação no sistema aqui estudado.

\section{CONSIDERAÇÕES FINAIS}

Os resultados desta pesquisa mostraram o estágio de institucionalização de NITs no sul do Brasil, que se caracteriza como sendo intermediário. Em face disso, evidencia-se a necessidade de aprofundamento daquele processo, que precisa ser deslocado rumo a um nível 
de institucionalização que viabilize aos NITs atuarem como interlocutores legítimos entre as ICTs e o setor privado para a difusão e comercialização da inovação.

Complementarmente, esta pesquisa evidencia também a complexidade da transferência de tecnologia no contexto brasileiro, e as formas que ICTs vêm adotando para participar de uma política e de programas de inovação, especialmente na região sul, objeto desta pesquisa. Teoricamente o estudo revela aspectos do processo de institucionalização a partir da noção de pilares institucionais, somada à questão das respostas organizacionais a ambientes institucionais complexos. Como se observou nas seções anteriores, a história dos NITs na região sul do país evidencia um processo de desenvolvimento da questão da relação entre IES e sociedade pautada em aspectos normativas, cultural-cognitivos mas, principalmente, regulativos.

As principais dificuldades evidenciadas naquele processo, principalmente vinculadas ao não surgimento de uma cultura empreendedora, à pouca autonomia e dificuldades para superação de entraves burocráticos na contratação de pessoal, parecem ser típicas daquele peculiar modelo de organização do campo. Aprofundar o conhecimento da teoria das organizações a respeito da relação entre os fundamentos, ou bases, sobre os quais ocorre o processo de institucionalização e as respostas organizacionais que emergem em decorrência das múltiplas pressões ambientais é uma via promissora de continuidade de investigação, no campo da inovação e em outros campos organizacionais e sociais.

Finalmente, como contribuição para outras ICTS, os resultados fornecem subsídio para ICTs que almejam implementar ou que estejam implementando NITS.

\section{REFERÊNCIAS}

ANG, S.; BENISCHKE, M.; DOH, J. The interactions of institutions on foreign market entry mode. Strategic Management Journal, New Jersey, v. 36, n. 10, p. 1536-1553, October 2015.

BATTILANA, J.; LECA, B. The role of resources in institutional entrepreneurship: insights for an approach to strategic management that combines agency and institution. In: CONSTANZO, L.; MACKAY, R. Handbook of research on strategy and foresight. Northampton: Edward Elgar Publishing, 2009. p. 260-274.

BAUER, M. W.; GASKELL, G. (Org.). Pesquisa qualitativa com texto, imagem e som: um manual prático. 6. ed. Petrópolis: Vozes, 2007.

BERRONE, P.; FOSFURI, A.; GELABERT, L.; GOMEZ-MEJIA, L. Necessity as the mother 
of 'green' inventions: institutional pressures and environmental innovations. Strategic Management Journal, New Jersey, v. 34, n. 8, p. 891-909, August 2013.

BERGER, P.; LUCKMANN, T. A construção social da realidade. Petrópolis: Vozes, 1998. BINDER, A. For love and money: organizations' creative responses to multiple environmental logics. Theory and Society, Amsterdam, v. 36, n. 6, p. 547-571, December 2007.

BRASIL. Lei n. ${ }^{\circ} 10.973$, de 2 de dezembro de 2004. Dispõe sobre incentivos à inovação e à pesquisa científica e tecnológica no ambiente produtivo e dá outras providências. Diário Oficial [da] República Federativa do Brasil, Brasília, DF, 3 dez. 2004. Disponível em: <http://www2.camara.leg.br/legin/fed/lei/2004/lei-10973-2-dezembro-2004-534975normaatualizada-pl.pdf>. Acesso em: 10 jun. 2015.

BRASIL. Lei n. ${ }^{\circ} 13.243$, de 11 de janeiro de 2016. Dispõe sobre estímulos ao desenvolvimento científico, à pesquisa, à capacitação científica e tecnológica e à inovação e altera a Lei $\mathrm{n}^{\circ} 10.973$, de 2 de dezembro de 2004, a Lei $\mathrm{n}^{\circ} 6.815$, de 19 de agosto de 1980, a Lei ${ }^{\circ} 8.666$, de 21 de junho de 1993, a Lei $n^{\circ} 12.462$, de 4 de agosto de 2011, a Lei $n^{\circ} 8.745$, de 9 de dezembro de 1993, a Lei no 8.958, de 20 de dezembro de 1994, a Lei no 8.010, de 29 de março de 1990, a Lei $n^{\circ}$ 8.032, de 12 de abril de 1990, e a Lei ${ }^{\circ} 12.772$, de 28 de dezembro de 2012, nos termos da Emenda Constitucional no 85, de 26 de fevereiro de 2015. Diário Oficial [da] República Federativa do Brasil, Brasília, DF, 12 jan. 2016. Disponível em: < http://www2.camara.leg.br/legin/fed/lei/2016/lei-13243-11-janeiro-2016-782239publicacaooriginal-149178-pl.html>. Acesso em: 10 jun. 2015.

BRASIL. MINISTÉRIO DA CIÊNCIA, TECNOLOGIA E INOVAÇÃO. Política de Propriedade Intelectual das Instituições Científicas e Tecnológicas do Brasil. Relatório Formict 2011. Brasília, 2012.

BRASIL. MINISTÉRIO DA CIÊNCIA, TECNOLOGIA E INOVAÇÃO. Política de Propriedade Intelectual das Instituições Científicas e Tecnológicas do Brasil. Relatório Formict 2012. Brasília, 2013.

BRASIL. MINISTÉRIO DA CIÊNCIA, TECNOLOGIA E INOVAÇÃO. Política de Propriedade Intelectual das Instituições Científicas e Tecnológicas do Brasil. Relatório Formict 2013. Brasília, 2014.

BRASIL. MINISTÉRIO DA CIÊNCIA, TECNOLOGIA E INOVAÇÃO. Política de Propriedade Intelectual das Instituições Científicas e Tecnológicas do Brasil. Relatório Formict 2014. Brasília, 2015. 
CALZOLAIO, A. E.; CORREIA, P.; DATHEIN, R. Sistema de inovação do Paraná: Análise do processo de inovação do Estado e implicações para política. Revista Economia \& Tecnologia, Curitiba, v. 9, n. 3, p. 55-72, Mar. 2013.

COOPER, D.; SCHINDLER, P. Métodos de pesquisa em Administração. Porto Alegre: Bookman, 2003.

CHANDLER, D.; HWANG, H. Learning From Learning Theory: A Model of Organizational Adoption Strategies at the Micro foundations of Institutional Theory. Journal of Management, Thousand Oaks, v. 41, n 5, p. 1446-1476, July 2015.

COLYVAS, J.; POWELL, W. Measures, metrics and myopia: the challenges and ramifications of sustaining academic entrepreneurship. Advances in the Study of Entrepreneurship, Innovation and Economic Growth, Bingley, v. 19, p. 79-111, 2009. COLYVAS, J. From divergent meanings to common practices: the early institutionalization of technology transfer in the life sciences at Stanford University. Research Policy, Amsterdam, v. 36, n. 4, p. 456-476, May 2007.

CRUBELLATE, J. Três contribuições conceituais neofuncionalistas à teoria institucional em organizações. Revista de Administração Contemporânea, Curitiba, v. 11, $1^{\text {a }}$ ed. esp., p. 199-222, 2007.

DIAS, J. C. Competências Organizacionais diferenciadoras em núcleos de inovação tecnológica. 2010. 209 f. Dissertação (Mestrado em Administração) - Universidade Federal do Paraná, Curitiba, 2010.

DIMAGGIO, P. J.; POWELL, W. W. A gaiola de ferro revisitada: isomorfismo institucional e racionalidade coletiva nos campos organizacionais. Revista de Administração de Empresa, São Paulo, v. 45, n. 2, p. 74-89, Abr-Jun 2005.

DORADO, S. Small groups as context for institutional entrepreneurship: an exploration of the emergence of commercial microfinance in Bolivia. Organization Studies, Thousand Oaks, v. 34, n. 4, p. 533-557, April 2013.

ETZKOWITZ, H.; LEYDESDORFF, L. The Dynamics of innovation: from National Systems and "Mode 2" to a Triple Helix of university-industry-government relations. Research Policy, Amsterdam, v. 29, n. 2, p. 109-123, February 2000.

FAULCONBRIDGE, J.; MUZIO, D. Global professional service firms and the challenge of institutional complexity: 'field relocation' as a response strategy. Journal of Management Studies, Medford, MA, v. 53, n. 1, p. 89-124, January 2016.

FORTEC. ASSOCIAÇÃO FÓRUM NACIONAL DE GESTORES DE INOVAÇÃO E TRANSFERÊNCIA DE TECNOLOGIA. Estatuto. Disponível em: <http://fortec.org.br/wp- 
content/uploads/2016/05/Estatuto-aprovado-em-151207-2.pdf>. Acesso em: 18 jun. 2015. FUENTELSAZ, L.; GARRIDO, E., MAICAS, J. Incumbents, technological change and institutions: how value of complementary resources varies across markets. Strategic Management Journal, New Jersey, v. 36, n. 12, p. 1778-1801, December 2015. GUARIDO FILHO, E. R. A construção da teoria institucional nos estudos organizacionais no Brasil: o período 1993-2007. 2008. 316 f. Tese (Doutorado em Administração) - Universidade Federal do Paraná, Curitiba, 2008.

GREENWOOD, R.; HININGS, C.; WHETTEN, D. Rethinking institutions and organizations. Journal of Management Studies, Medford, MA, v. 51, n. 7, p. 1206-1220, November 2014. GREENWOOD, R.; RAYNARD, M.; KODEIH, F., MICELOTTA, E., LOUNSBURY, M. Institutional complexity and organizational responses. Academy of Management Annals, Bryarcliff Manor, NY, v. 5, n. 1, p. 317-371, June 2011.

HAUNSCHILD, P.; CHANDLER, D. Institutional-level learning: learning as a source of institutional change. In: GREENWOOD, R. et al. (Orgs). The Sage Handbook of Organizational Institutionalism. London: Sage, 2008, p. 624-649. HENFRIDSSON, O.; YOO, Y. The liminality of trajectory shifts in institutional entrepreneurship. Organization Science, Catonsville, v. 25, n. 3, p. 932-950, May-June 2014. HOLMES, R.M.; MILLER T.; HITT, M.A.; SALMADOR, M.P. The interrelationships among informal institutions, formal institutions, and inward foreign direct investment. Journal of Management, Thousand Oaks, v. 39, n. 2, p. 531-566, February 2013. LOBATO, A. et al. Produção do conhecimento tecnológico na UFMG. Perspectivas em Ciência da Informação, v. 5, n. 2, p. 231-242, Jul.-Dez. 2000.

LOTUFO, R. A. A. Institucionalização de Núcleos de Inovação Tecnológica e a Experiência da Inova Unicamp. In: SANTOS, M. E. R. dos; TOLEDO, P. T. M. de; LOTUFO, R. de A. (Org.) Transferência de Tecnologia: estratégias para a estruturação e gestão de Núcleos de Inovação Tecnológica. Campinas: Komedi, 2009, p.41-74.

MACHADO-DA-SILVA, C.; FONSECA, V.; CRUBELLATE, J. Estrutura, agência e interpretação: elementos para uma abordagem recursiva do processo de institucionalização.

Revista de Administração Contemporânea, Curitiba, v. 9, $1^{\text {a }}$ Edição Especial, p. 9-39, JanJun 2005.

MIRABEAU, L.; MIGUIRE, S. From autonomous strategic behavior to emergent strategy. Strategic Management Journal, New Jersey, v. 35, n. 8, p. 1202-1229, August 2014. OLIVEIRA, P.; MACHADO-DA-SILVA, C. Características culturais nacionais em organizações industriais no setor alimentício paranaense. Revista Organizações \& 
Sociedade, Salvador, v.8, n. 22, p.1-20, Set-Dez 2001.

OLIVER, C. Strategic responses to institutional processes. Academy of Management Review, Bryarcliff Manor, NY, v. 16, n.1, p. 145-179, Jan-Mar 1991.

PIRES, E. A.: QUINTELLA, C. M. A. T. Política de Propriedade Intelectual e Transferência de Tecnologia nasUniversidades: uma perspectiva do NIT da Universidade Federal do Recôncavo da Bahia. Holos, Natal, v. 6, n. 31, p. 178-195, 2015.

QUATTRONE, P. Governing social orders, unfolding rationality, and Jesuit accounting practices: a procedural approach to institutional logics. Administrative Science Quarterly, Ithaca, v. 60, n. 3, p. 411-445, September 2015.

RAAIJMAKERS, A.; VERMEULEN, P.; MEEUS, M.; ZIETSMA, C. I need time! Exploring pathways to compliance under institutional complexity. Academy of Management Journal, Bryarcliff Manor, v. 58, n. 1, p. 85-110, February 2015.

RAUEN, C. V. O Novo Marco Legal da Inovação no Brasil: O que muda na Relação ICTEmpresa? Radar, v. 43, p.21-35, 2016. Disponível em:

<http://repositorio.ipea.gov.br/bitstream/11058/6051/1/Radar_n43_novo.pdf>. Acesso em: 18 jun. 2015.

RODRIGUES, F.; GAVA, R. Capacidade de Apoio à Inovação dos Institutos Federais e das Universidades Federais no Estado de Minas Gerais: Um estudo comparativo. REAd, Porto Alegre, v. 22, n. 1, p. 26-51, Jan-Abr 2016.

SAKA-HELMHOUT, A.; DEEG, R.; GREENWOOD, R. The MNE as a Challenge to Institutional Theory: Key Concepts, Recent Developments and Empirical Evidence. Journal of Management Studies, Medford, MA, v. 53, n.1, p. 1-11, January 2016.

SCHERER, A.; PALAZZO, G.; SEIDL, D. Managing Legitimacy in Complex and Heterogeneous Environments: Sustainable Development in a Globalized World. Journal of Management Studies, Medford, MA, v. 50, n. 2, p. 259-284, March 2013.

SCOTT, W. Institutions and organizations. 2. ed. Thousand Oaks: Sage, 2001.

SMETS, M.; MORRIS, T.; GREENWOOD, R. From practice to field: a multilevel model of practice-driven institutional change. Academy of Management Journal, Bryarcliff Manor, v. 5, n. 4, p. 877-904, August 2012.

STEVENS, C.; XIE, E.; PENG, M. Toward a legitimacy-based view of political risk: the case of Google in China. Strategic Management Journal, New Jersey, v. 37, n. 5, May 2015. TOLBERT, P.; ZUCKER, L. A institucionalização da teoria institucional. In: CLEGG, S.; HARDY, C.; NORD, W. (Orgs.). Handbook de estudos organizacionais: modelos de análise e novas questões em estudos organizacionais. São Paulo: Atlas, 1998. p. 196-219. 
TOLBERT, P.; DAVID, R.; SINE, W. Studying choice and change: the intersection of institutional theory and entrepreneurship research. Organization Science, Catonsville, v. 22, n. 5, p. 1332-1344, Sep-Oct 2011.

TOLEDO, P. T. M. de. A gestão estratégica dos Núcleos de Inovação Tecnológica: cenários, desafios e perspectivas. In: SANTOS, M. E. R. dos; TOLEDO, P. T. M. de; LOTUFO, R. de A. (Orgs.). Transferência de Tecnologia: estratégias para estruturação e gestão dos Núcleos de Inovação Tecnológica. Campinas: Komedi, 2009, p. 109-166.

TORKOMIAN, A. L. V. Panorama dos Núcleos de Inovação Tecnológica no Brasil. In: SANTOS, M. E. R. dos; TOLEDO, P. T. M. de; LOTUFO, R. de A. (Orgs.).Transferência de Tecnologia: estratégias para estruturação e gestão dos Núcleos de Inovação Tecnológica. Campinas: Komedi, 2009, p. 21-37.

VORONOV, M.; DE CLERCQ, D.; HININGS, C. R. Conformity and distinctiveness in a global institutional framework: the legitimation of Ontario Fine Wine. Journal of Management Studies, Medford, MA, v. 50, n. 4, p. 607-645, June 2013.

WALKER, K.; SCHLOSSER, F.; DEEPHOUSE, D. Organizational ingenuity and the paradox of embedded agency: the case of the embryonic Ontario solar energy industry. Organization Studies, Thousand Oaks, v. 35, n. 4, p. 613-634, April 2014 ZUCKER, L. The role of institutionalization in cultural persistence. American Sociology Review, Washington, DC, v. 42, n. 5, p.726-743, October 1977.

ZUCKER, L. G. Institutional theories of organization. Annual Review of Sociology, Palo Alto, v. 13, p. 443-464, Jan-Dec 1987. 